\title{
Aspect based Sentiment Analysis of Employee's Review Experience
}

\author{
Nasa Zata Dina ${ }^{1)^{*}}$, Nyoman Juniarta ${ }^{2)}$ \\ ${ }^{1)}$ Department of Engineering, Faculty of Vocational Studies, Universitas Airlangga, Indonesia \\ B Campus, Universitas Airlangga, Jalan Dharmawangsa Dalam, 60268, Surabaya \\ ${ }^{1)}$ nasazatadina@vokasi.unair.ac.id \\ ${ }^{2)}$ Department of Informatics, Faculty of IAEM (Informatique, Automatique, Électronique-Électrotechnique, et Mécanique), \\ Université de Lorraine, France \\ 34 Cours Léopold, 54000, Nancy \\ ${ }^{2)}$ nyoman.juniarta@univ-lorraine.fr
}

\author{
Article history: \\ Received 4 March 2020 \\ Revised 16 April 2020 \\ Accepted 21 April 2020 \\ Available online 28 April 2020
}

Keywords:

Decision Tree

Sentiment analysis

Support Vector Machine

Text mining

Word list

\begin{abstract}
Background: Employees of technology companies evaluate their experience through online reviews. Online reviews of companies from employees or former employees help job seeker to find out the weaknesses and strengths of the companies. The reviews can be used as an evaluation tool for each technology company to understand their employee's perceptions. However, most information on online reviews is not well responded since some of the detailed information of the company is missing.

Objective: This study aims to generate an Aspect-based Sentiment Analysis using user review data. The review data were then extracted and classified into five aspects: work balance, culture value, career opportunities, company benefit, and management. The output of this study is the aspect score from each company.

Methods: This study suggests a method to analyze online reviews from employees in detail, so it can prevent the missing of specific information. The analysis was sequentially carried out in five stages. First, user review data were crawled from Glassdoor and stored in a database. Second, the raw data were processed in the data pre-processing stage to delete the incomplete data. Third, the words other than noun keyword were eliminated using Standford POS Tagger. Fourth, the noun keywords were then classified into each aspect. Finally, the aspect score was calculated based on the aspect-based sentiment analysis.

Results: Result showed that the proposed method managed to turn raw review data into five aspects based on user perception.

Conclusion: The study provides information for two parties, job seeker and the company. The analysis of the review could help the job seeker to decide which company that suits his need and ability. For the companies, it can be a great assistance because they will be more aware of their strengths and weaknesses. This study could possibly also provide ratings to the companies based on the aspects that have been determined.
\end{abstract}

\section{INTRODUCTION}

Human Resources (HR) Manager has many important tasks in a company. The tasks include formatting the employee's recruitment, planning the employee's training and orientation, and evaluating the employee's satisfaction. HR Manager is a crucial position because HR Manager becomes a liaison between the management and employees. HR Manager has to understand the employee's perception of the company. One way to honestly determine employee's perceptions of the company is to frequently conduct surveys. Recently, employees often write their honest reviews on a platform called Glassdoor. Glassdoor is often called as a crowd sourced employer branding platform [1]. Everyone, which comes from a specific company, can give a rating and also write their

\footnotetext{
${ }^{*}$ Corresponding author
} 
work experience at the company where they work. The reviews can be very helpful for the readers. They could compare the advantages and disadvantages of each work experience. The background of the readers varies from a job seeker, employee, and even HR Manager. The information extracted from the platform such as Glassdoor was classified as an honest opinion because the employee posted their review without anyone asking. The HR Manager can utilize the review data so that the HR Manager can easily find out the employee's perception. The biggest challenge is a large amount of unstructured review data. Also, the review data from Glassdoor are not freely accessible, so it requires more effort to obtain the large review data.

In this study, we focused on Aspect-based Sentiment Analysis. We propose an extension of Liu's Aspect-based Sentiment Analysis methodology to be applied to the crowd sourced employer branding domain, especially on the Glassdoor platform. The existing works of Aspect-based Sentiment Analysis mostly focus on product review mining. For example, Liu et al [2] and Shahkhali et al [3] used various camera product reviews, Sindhu [4] summarized a lot of product reviews on Amazon, and Shrividya et al [5] have considered reviews on iPod product. The users' comment in a product review is usually predictable because the features have already been mentioned, and the review itself is all about whether they like the features or not. Recently, the focus of this Aspect-based Sentiment Analysis research began to use a lot of data on tourism products. Akhtar et al [6] used hotel review from TripAdvisor platform to build hotel recommender system, Al-Smadi [7] enhanced AspectBased Sentiment Analysis from Arabic's hotel only while Garcia-Pablos et al [8] use various data from several domains like hotels, restaurants, electronic devices in different languages such as English, Spanish, French and Dutch to classify users' review using unsupervised approaches for Aspect-based Sentiment Analysis.

Marrese-Taylor et al [1] stated that there is a difference between usual product review and tourism product review. The users tend to write a longer review of tourism product because they describe their feeling towards it. The users often use complex sentences to describe the tourism product. So, they concluded that a different product produces different reviews, and it has diverse characteristics [1]. Since product review and tourism product review have become a trend a few years back in Aspect-based Sentiment Analysis research, we decided to do research in crowd sourced employer branding domain. The most important reason to choose crowd sourced employer branding domain is because, to the best of our knowledge, the existing approaches have not addressed the issues in this domain yet. Hence in this study, we proposed an Aspect-based Sentiment Analysis to generate a lot of insights from crowd sourced data in Glassdoor platform, so the company as an employer can easily find out the employee's perception and create employer branding strategies.

The rest of this paper is structured as follows. In section II, we discuss methods and give the data description and research procedure. In section III, we write the result of the experiments which is followed by section IV where we discuss the explanation of the results and in-depth analysis. Finally, in section V, we draw main conclusions and propose future work.

\section{METHODS}

\section{A. Data description}

We collected our study data review at www.glassdoor.com. Glassdoor is a site where current and former employees review companies and their management anonymously [9]. Fig. 1 shows a screen shot of Glassdoor.com. In this study, we used data from three big technology companies: Amazon, Google, and Apple. The number of reviews from data collection is 27820 reviews, and it consists of seven attributes: company's name, location, dates, job-title, summary, pros, cons, and overall ratings. The first step of the study was to extract three out of seven attributes. They are company's name, summary, and overall rating attributes only. The dataset that has been obtained is saved to the following link http://tiny.cc/reviewdataGD

TABLE 1

THE NUMBER OF DATASET FROM EACH COMPANY

\begin{tabular}{cc}
\hline THE NUMBER OF DATASET FROM EACH COMPANY \\
\hline Dataset from the company & The number of the data \\
\hline Amazon & 10000 \\
Apple & 10000 \\
Google & 7820 \\
Total & 27820 \\
\hline
\end{tabular}




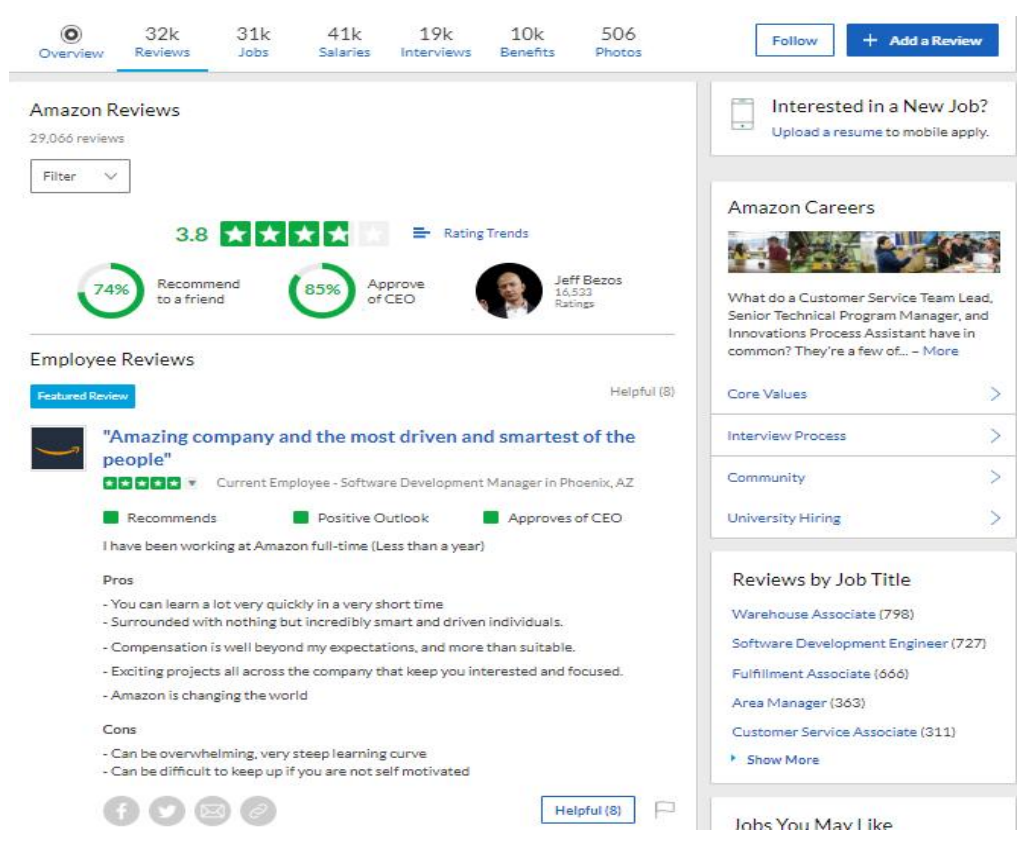

Fig. 1 Glassdoor.com

\section{B. Research Procedure}

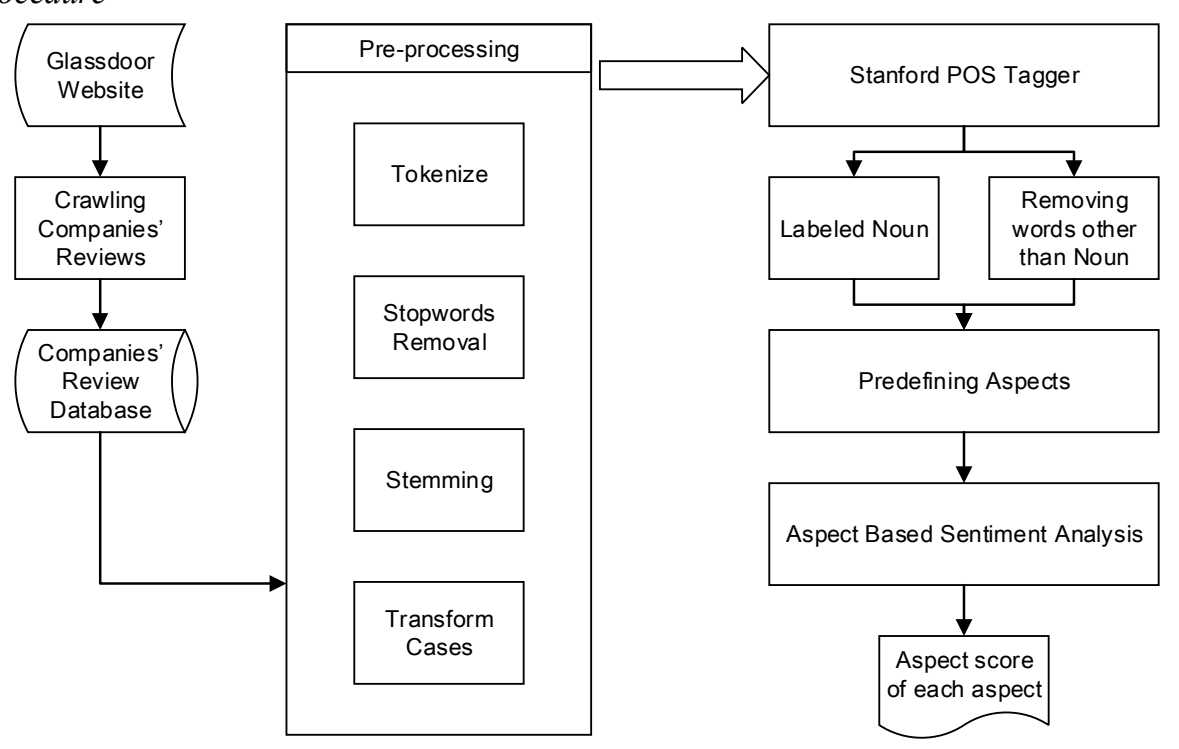

Fig. 2 Research procedure

The research procedure is illustrated in Fig. 3. It consisted of five steps as follows:

1. Crawling companies' review

As the latest company's review dataset was not available, then the data from Glassdoor website were crawled using Webharvy Web Scrapper [10]. Fig. 2 shows a screenshot of Webharvy. It took an input of the URL of the company's webpage on the Glassdoor site, and then chose the attributes which were crawled using a feature in Webharvey. In this study, the company's name, dates, job title, summary, pros, cons, and overall ratings were retrieved. Then, after all the pages had been crawled, the dataset was exported into a CSV file. The same steps were used to retrieve the data from other companies. Therefore, there were three CSV files from the three 
companies. Later, the CSV files were converted to excel files. Table 2 represents the example of the dataset from each company with seven attributes.

TABLE 2

THE EXAMPLE OF THE DATASET

\begin{tabular}{|c|c|c|c|c|c|c|}
\hline Company's Name & Date & Job title & Summary & Pros & Cons & Overall ratings \\
\hline Amazon & Feb 3, 2017 & $\begin{array}{l}\text { Current } \\
\text { Employee - } \\
\text { Picker }\end{array}$ & $\begin{array}{l}\text { I'm extremely } \\
\text { fond of this Job! } \\
\text { You have to } \\
\text { Make this Job } \\
\text { Your focus and } \\
\text { work Your Life } \\
\text { Around it. }\end{array}$ & $\begin{array}{l}\text { The pay is Great. } \\
\text { Peak season is } \\
\text { the best to work. } \\
\text { They offer Great } \\
\text { Benefits. Full } \\
\text { Amazonians } \\
\text { Receive Stocks } \\
\text { and Monthly } \\
\text { Bonuses. }\end{array}$ & $\begin{array}{l}\text { There are Long } \\
\text { shifts about } 11 \\
\text { hours. Once You } \\
\text { Use all Your time } \\
\text { You are } \\
\text { Terminated no } \\
\text { room for } \\
\text { discussion. If You } \\
\text { are Hostile You } \\
\text { will Be terminated. }\end{array}$ & 4 \\
\hline Apple & $\begin{array}{l}\text { Dec 11, } \\
2018\end{array}$ & $\begin{array}{l}\text { Former } \\
\text { Employee - } \\
\text { Systems Design } \\
\text { Engineer }\end{array}$ & $\begin{array}{l}\text { Great place to } \\
\text { work and gain } \\
\text { experience }\end{array}$ & $\begin{array}{l}\text { Good benefits, } \\
\text { very good } \\
\text { experience, } \\
\text { recognizable } \\
\text { name. }\end{array}$ & $\begin{array}{l}\text { Somewhat lower } \\
\text { pay, difficult to } \\
\text { advance in career, } \\
\text { imbalanced work } \\
\text { life. }\end{array}$ & 4 \\
\hline Google & Dec 1, 2018 & $\begin{array}{l}\text { Former } \\
\text { Employee - } \\
\text { Anonymous } \\
\text { Employee }\end{array}$ & $\begin{array}{l}\text { Great Company } \\
\text { With No Down } \\
\text { Sides }\end{array}$ & $\begin{array}{l}\text { Really fun work } \\
\text { environment with } \\
\text { startup }\end{array}$ & $\begin{array}{l}\text { Really no down } \\
\text { sides that I saw. }\end{array}$ & 5 \\
\hline
\end{tabular}

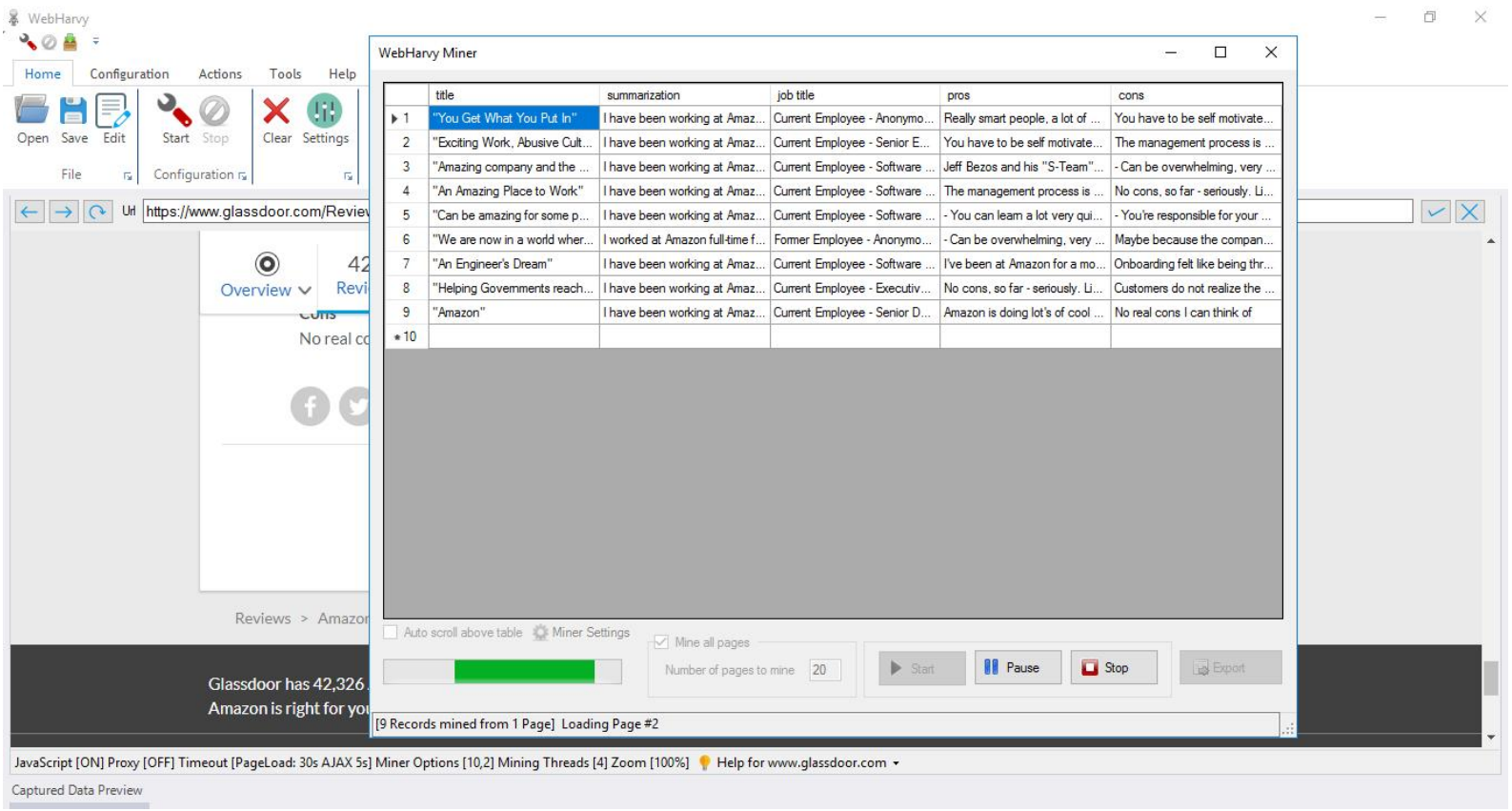

Fig. 3 Crawling the dataset using Webharvy Web Scrapper

\section{2. $\quad$ Data pre-processing}

Data pre-processing is a process to convert raw data into formatted data so that the computer can understand and read the data easily. The general steps of data pre-processing are incomplete data deletion, data selection, data integration and data formatting [11]. Data pre-processing has to be done because the quality of the dataset determines the result. The main objectives of data pre-processing are summarized as follows: (1) data cleansing; (2) data integration; (3) data transformation; (4) data reduction; (5) data discretization. For data pre-processing in this study, the tool called RapidMiner was used. RapidMiner is an open source software for processing data, text mining, and predictive analysis [12]. The number of the raw data is not limited in RapidMiner, and the type of the raw data can vary including databases and text [13]. RapidMiner's visualization is easily understandable, and there are also 
many additional extensions to fully use the features. For data pre-processing, there are four steps that must be done. The visualization of the four steps data pre-processing in RapidMiner is shown in Fig. 3. The explanation of each step as follows:

a. Tokenize

Tokenize is an operator for splitting the sentence in the document into a sequence of words [14]. The purpose of this sub process is to separate words from a document, so this list of words can be used for the next sub process.

b. Stop words removal

Stop words are commonly known as a list of words that often appear in the sentences. They often appear because stop words include prepositions, articles, and pronouns [14]. The stop words should be eliminated because they will be taking up space in the database, but they do not provide any useful information.

c. Stemming

Stemming is the process of mapping and breaking down the form of a word into its basic word form. Simply put, it is the process of changing suffixes words into basic words. The stemmer used in this study was Porter Stemmer [15]. Porter Stemmer removes suffixes from English words. Porter stemmer is well known of its less error rate than the other stemmers such as Lovins Stemmer, Paice/Husk Stemmer, and Dawson Stemmer [16].

d. Transform cases

Transforms case is an operator which converts all characters or texts in a document into all lower case or all upper case. It is done to avoid the confusion of words [17].

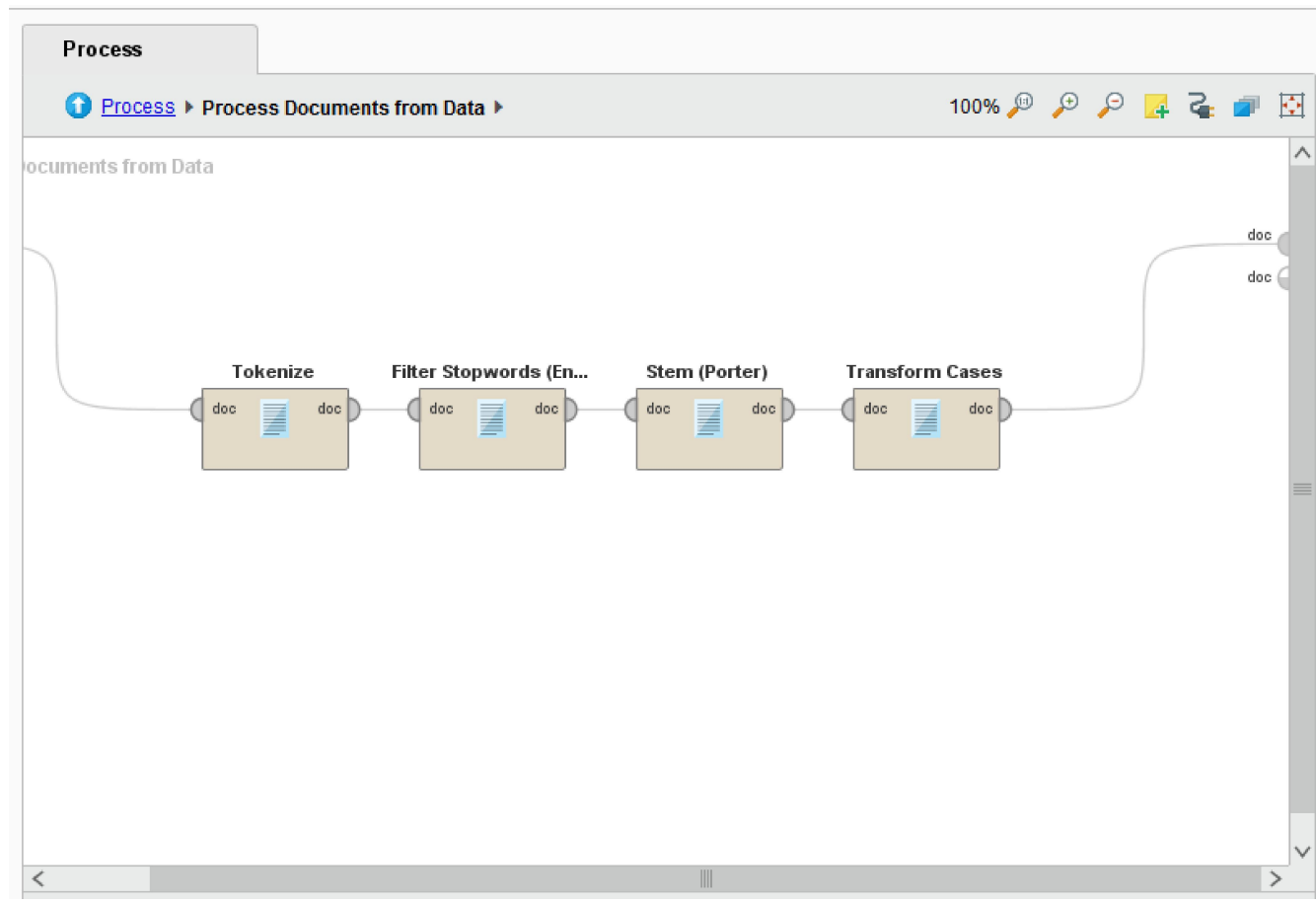

Fig. 4 The visualization of data pre-processing in RapidMiner

3. Labeled noun using Stanford POS tagger

The output of the data pre-processing which is a list of keywords is used as an input in this stage. All of the keywords were inserted and checked using Stanford POS tagger software. The previous work has been done by Yi et al in 2003. They used Stanford POS tagger to label the keywords based on their part of speech [18]. The POS tagger determines which part of speech of each keyword. The 36 tags of part of speech from Stanford POS tagger can be seen at Table 3. In this paper, keywords that are tagged as nouns are extracted. 
Table 3 shows NN, NNS, NNP, and NNP are nouns. The output of this stage is a list of noun tagged keywords.

TABLE 3

TAG AND DESCRIPTION FROM STANFORD POS TAGGER

\begin{tabular}{ll}
\hline Tag & Description \\
\hline CC & Coordinating conjunction \\
CD & Cardinal number \\
DT & Determiner \\
EX & Existential there \\
FW & Foreign word \\
IN & Preposition or subordinating conjunction \\
JJ & Adjective \\
JJR & Adjective, comparative \\
JJS & Adjective, superlative \\
LS & List item marker \\
MD & Modal \\
NN & Noun, singular or mass \\
NNS & Noun, plural \\
NNP & Proper noun, singular \\
NNPS & Proper noun, plural \\
PDT & Predeterminer \\
POS & Possessive ending \\
PRP & Personal pronoun \\
PRP\$ & Possessive pronoun \\
RB & Adverb \\
RBR & Adverb, comparative \\
RBS & Adverb, superlative \\
RP & Particle \\
SYM & Symbol \\
TO & To \\
UH & Interjection \\
VB & Verb, base form \\
VBD & Verb, past tense \\
VBG & Verb, gerund or present participle \\
VBN & Verb, past participle \\
VBP & Verb, non-3rd person singular present \\
VBZ & Verb, 3rd person singular present \\
WDT & Wh-determiner \\
WP & Wh-pronoun \\
WP\$ & Possessive wh-pronoun \\
WRB & Wh-adverb \\
\hline & \\
\hline
\end{tabular}

4. $\quad$ Predefining aspects

On this stage, the linguists categorize the keywords into some of the predefined categories. The predefined categories are commonly called as aspects. These aspects are collections of keywords that often appear in the data [6].

5. Aspect-based Sentiment Analysis

Aspect-based Sentiment Analysis is used to determine the score of each aspect [6]. The each aspect has a list of keywords and the term of frequency (TF) of each keyword that will be checked. The sentiment analysis of each document which has the keyword is given the score. The score is given for negative and positive sentiment analyses. The labels of negative and positive sentiment are based on the overall rating value. If the overall rating value is more than 2, then it is labeled as a positive sentiment. However, if the overall rating value is less than equal to 2 , then it is labeled as negative sentiment. The example of overall rating value can be seen in Table 2 column number 7 .

\section{RESULTS}

Table 4 shows the example of the keywords that have high TF in the review dataset from three companies. Thus, these keywords are the words that appear most often in the data set. After the list of keyword was determined, it was then categorized based on five aspects. The five aspects were proposed by Dabirian et al [19]. He found that those 
aspects which were often called as employer branding value proposition were the things that employees care most in evaluating places to work. Those five aspects were "work balance", "culture values", "career opportunities", "company benefit" and "management".

TABLE 4

THE EXAMPLE OF MANUALLY DEFINED ASPECTS AND THEIR FREQUENTLY OCCURRING KEYWORDS

\begin{tabular}{|c|c|c|c|c|}
\hline Work balance & Culture values & Career opportunities & Company benefit & Management \\
\hline Overwork & Vision & Interview & Location & Figure \\
\hline Risk & Motive & Question & Cost & Manager \\
\hline Space & Active & Step & Deal & Procurement \\
\hline \multirow{12}{*}{ Loss } & Dedication & Application & Ride & Micromanagement \\
\hline & Plan & Decline & House & Share \\
\hline & Pleasure & Choice & Gain & User \\
\hline & Rule & Offer & Navigate & Boss \\
\hline & Truth & Gain & Campus & \\
\hline & Fine & Inexperience & View & \\
\hline & Flaw & Prospect & & \\
\hline & Inconsistent & Income & & \\
\hline & Integrity & Cost & & \\
\hline & Teamwork & & & \\
\hline & Inform & & & \\
\hline & Exhilaration & & & \\
\hline
\end{tabular}

The score was given for each aspect based on the overall rating value as explained in section II sub section 5 . The value was calculated as a positive or negative score. The results were illustrated using bar chart. The $\mathrm{x}$-coordinate describes the aspects while the y-coordinate shows the number of positive and negative scores. Each bar chart shows the aspects' score from each company. The aspects' score from Amazon, Apple and Google can be seen at Fig. 5, Fig. 6 and Fig. 7 respectively. From the figure, we can conclude that each company has its strength and its weakness. Amazon has its positive score higher than the negative score on every aspect while Apple has the highest positive score in career opportunities. The chart of Google review is almost similar to Amazon, but the difference that makes Google better than Amazon is the negative score of culture values and management aspects which were zero. It means that there are no negative reviews from those aspects. To clarify the result, Table 5 shows in detail the keywords of company benefit's aspect from Google. From Table 5 which is the information from the keywords, it obtained 32 positive score and four negative score. The result of these aspects helps to identify the weaker aspect of the company based on the employee and former employee review.

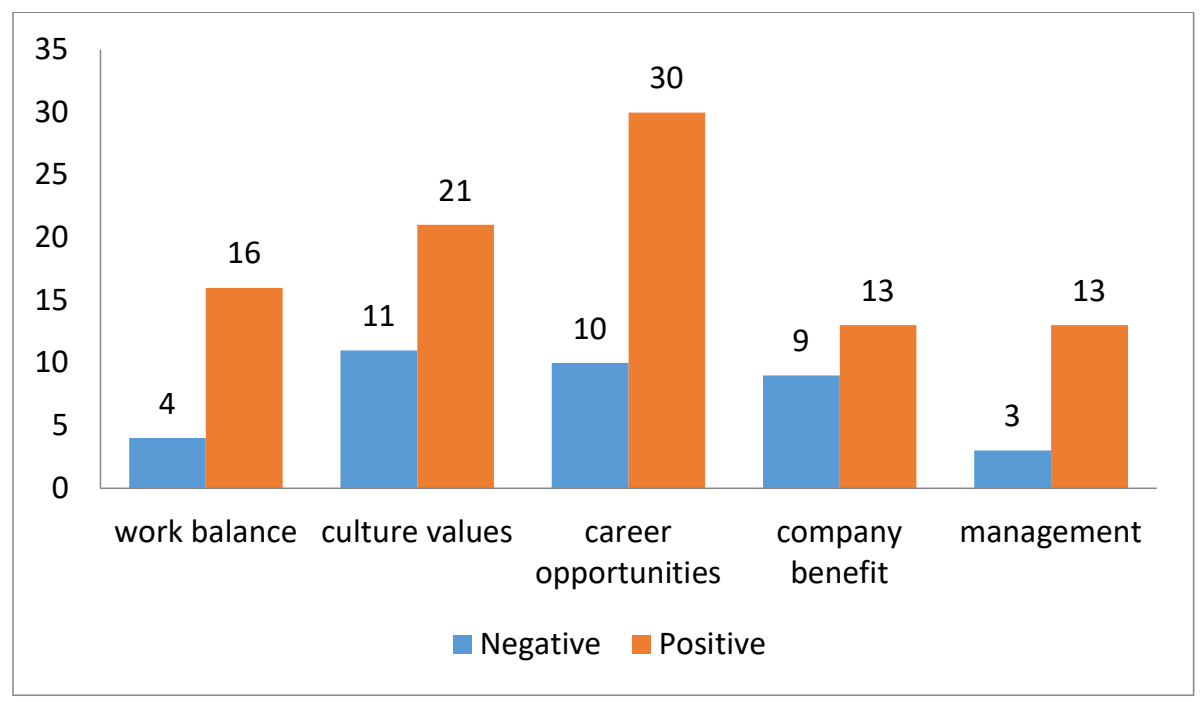

Fig. 5 Aspect score of each aspect from Amazon company's review 


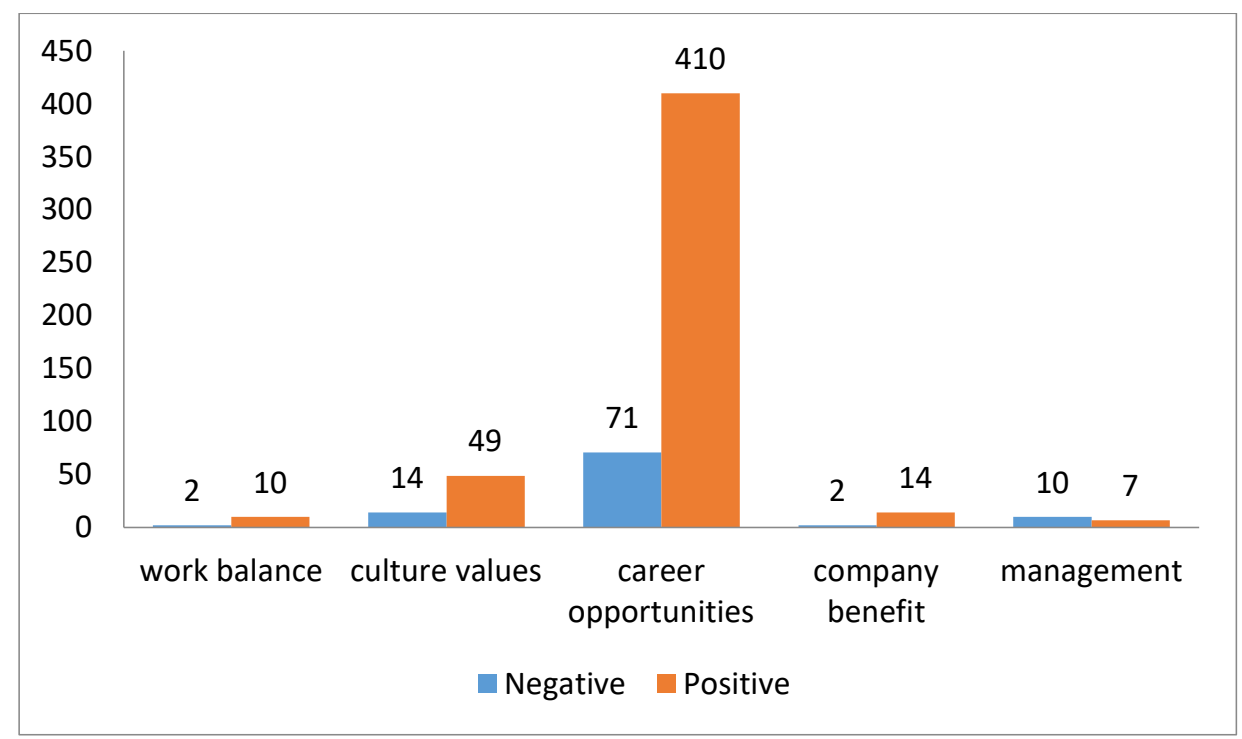

Fig. 6 Aspect score of each aspect from Apple company's review

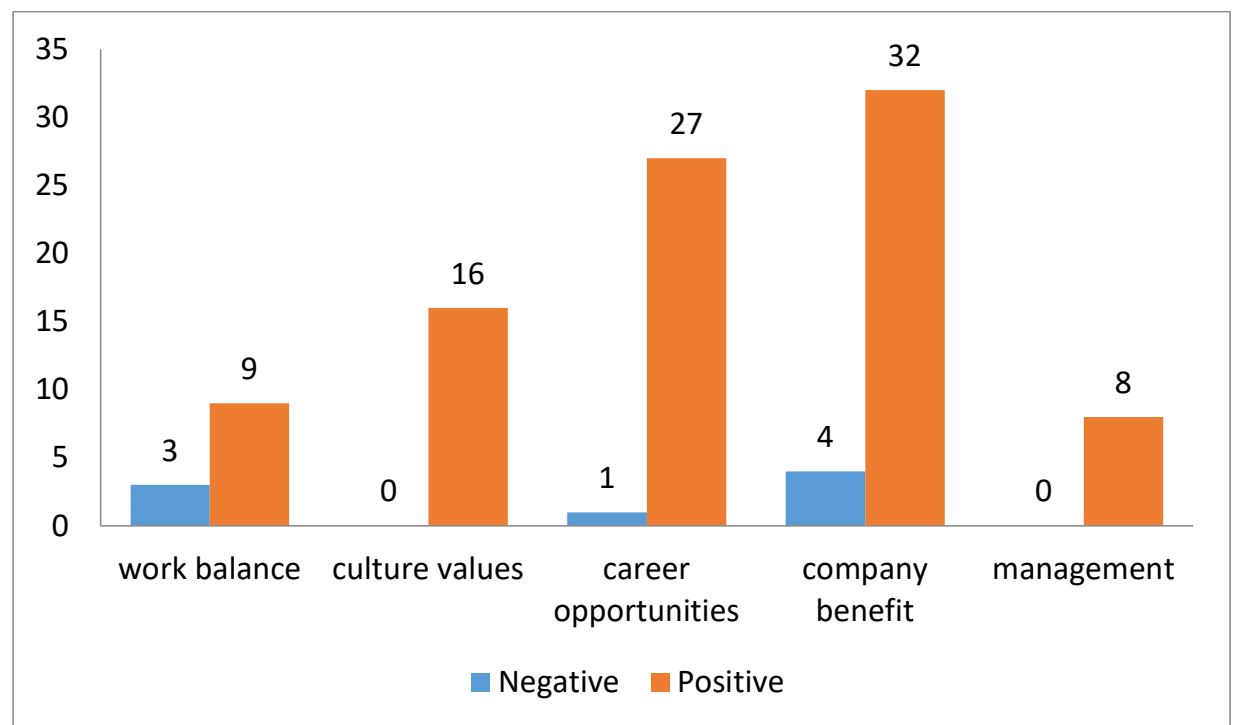

Fig. 7 Aspect score of each aspect from Google company’s review

TABLE 5

THE EXAMPLE OF KEYWORDS FROM COMPANY BENEFIT ASPECT AND EACH SCORE

\begin{tabular}{lrr}
\hline Keywords & Positive Score & \multicolumn{2}{c}{ Negative Score } \\
\hline Location & 4 & 0 \\
Cost & 3 & 1 \\
Deal & 4 & 0 \\
Ride & 4 & 0 \\
House & 4 & 3 \\
Gain & 4 & 0 \\
Navigate & 4 & 0 \\
Campus & 4 & 0 \\
Total Score & 32 & 4 \\
\hline
\end{tabular}

In order to indicate the accuracy of this study, the evaluation of aspect based sentiment analysis for three companies is shown in Table 6. Table 6 describes the precision and recall of sentiment analysis result for each aspect per company. Precision is the level of accuracy between the information requested by the user and the 
answers given by the system. Recall is the level of success of the system in rediscovering information. Overall the precision values in Table 6 are mostly less than recall values, it means that the system successfully rediscovers the information but the answer given from the system is rarely answered correctly by the system. Liu stated in [2] that there is a possibility that many sentences are not opinionated sentences in the review data so that the precision value is lower than the recall value.

TABLE 6

PERFORMANCE RESULT

\begin{tabular}{lcccccc}
\hline \multirow{1}{*}{ Aspects } & \multicolumn{2}{c}{ Amazon Company } & \multicolumn{2}{c}{ Apple Company } & \multicolumn{2}{c}{ Google Company } \\
\cline { 2 - 6 } & Precision & Recall & Precision & Recall & Precision & Recall \\
Work Balance & 64.28 & 90 & 69.23 & 69.23 & 85.71 & 85.7 \\
Culture Value & 68.42 & 86.66 & 80 & 92.3 & 85.18 & 95.8 \\
Career Opportunities & 62.16 & 92 & 66.21 & 96 & 64 & 94.11 \\
Company Benefit & 70.37 & 86.36 & $75 \%$ & 95.7 & 86.67 & 92.85 \\
Management & 63.15 & 92.3 & 54.5 & 85.7 & 72.72 & 88.8 \\
\hline
\end{tabular}

\section{DISCUSSION}

The analysis and summarization of user reviews of three giant companies in Glassdoor are the main focus of this study. The user reviews often provided valuable information about the weaknesses and strengths when working for a particular company. To find out the opinion from the current and former employees, the analysis of the review has been done. Data pre-processing has been done to avoid unwanted data on raw review data. Srividyaet al [5] applied POS Tagger to find the noun phrase because aspects are always in the noun phrase form. Thus, we directly did POS Tagger to verify the noun words without considering another POS such as adjectives, adverbs, etc. Akhtar [6] also confirms that aspect term commonly refers to the word that belongs to a noun phrase.

The frequent keywords and its aspect score are provided as important information to support the companies' claim about their workspace. The results of this study can be seen in Fig 5-7. Each company has its aspect score. The aspect score implies which of these aspects of the company under the study are better than the other company. The analysis of user review is different from the star rating on Glassdoor. It could be more realistic than the one provided by star rating. The user is only given five choices: 1 to 5 . The bigger number means the better while DucHong Pham et al [20] combined both aspect scores as they called aspect weight and aspect rating to generate the overall rating. Duc-hong [20] specifically conducted their experiment using user review data from a hotel domain. Considering the performance result, a method that can identify specific sentences must be involved because there are some issues related to a lot of non-opinionated sentences in the review data. It causes poor precision value. Linguist involvement is still inseparable as verifiers of specific sentences.

The study provides information for two parties, job seeker and the company. The analysis of the review could help the job seeker to decide which company that suits his need and ability. For the companies, it should be a great help because they will be more aware about their strong and weak aspects. This study could possibly also provide ratings to the companies based on the aspects that have been determined, such as rating to the company based on the aspect of career opportunities. Bafna et al [21] implements a dynamic system, so during a period of time, user reviews automatically extracted and updated certain aspect by grouping synonyms nouns, but their study was limited by product review only. The limitation of this study is we could not distinguish whether the review is honest or just wants to bring down certain companies. However, since the platform tightened the terms of use at any time, it could minimize the risk.

\section{V.CONCLUSIONS}

This study provides a way to extract employee review on predetermined platform called Glassdoor. An Aspectbased Sentiment Analysis managed to calculate the aspect score based on the number of frequent keywords on the review. It was started by crawling the review data from Glassdoor using Webharvy Web Scrapper, categorizing the unprocessed raw data of the user review, and storing them in the database. RapidMiner was used to do preprocessing on raw review data and produced keywords. The keywords were calculated based its term frequency. Next, only nouns were extracted from the group of keywords using Standford POS Tagger. Nouns were then classified based on their aspects. Last, aspect score was counted and displayed in a graphical form. 


\section{REFERENCES}

[1] E. Marrese-Taylor, J. D. Vel'asquez, F. Bravo-Marquez, and Y. Matsuo, "Identifying Customer Preferences about Tourism Products using an Aspect-Based Opinion Mining Approach", in 17th International Conference in Knowledge Based and Intelligent Information and Engineering Systems, Kitakyushu, Japan, September 2013, pp. 182-191.

[2] M. Hu, B. Liu, "Mining opinion features in customer reviews", in Proceedings of the National Conference on Artificial Intelligence, Menlo Park, CA, MIT Press, 2004, pp. 755-760, 2004.

[3] M. A. Shahkhali, F. Ahmadi-Abkenari, "Sentiment Mining on Products Features based on Part of Speech Tagging Approach", International journal of Computer Science \& Network Solutions, vol. 3, no. 12, pp. 1-12, 2015.

[4] C. S. Sindhu, "Aspect Based Sentiment Analysis of Amazon Product Reviews", International Journal of Pure and Applied Mathematics, vol. 118, no. 22, pp. 151-157, 2014.

[5] K. Srividya, A. M. Sowjanya, "Aspect Based Sentiment Analysis using POS Tagging and TFIDF ", International Journal of Engineering and Advanced Technology, vol. 8, no. 6, pp. 1960-1963, 2019

[6] M. S. Akhtar, D. Gupta, A. Ekbal, P. Bhattacharyya, "Feature selection and ensemble construction: a two-step method for aspect based sentiment analysis", Knowledge-Based System, vol. 125, pp. 116-135, 2017.

[7] M. Al-Smadi, O. Qawasmeh, M. Al-Ayyoub, Y. Jararweh, B. Gupta, "Deep Recurrent neural network vs. support vector machine for aspect-based sentiment analysis of Arabic hotels' reviews", Journal of Computational Science, vol. 27, pp. 386-393, 2018.

[8] A. Garcia-Pablos, M. Cuadros, G. Rigau, "W2VLDA: Almost unsupervised system for Aspect Based Sentiment Analysis", Expert Systems with Applications, vol. 91, pp. 127-137, 2018.

[9] Y. Al-Amrani, M. Lazaar, K.E. Elkadiri, "Sentiment Analysis using supervised classification algorithms", in Proceedings of the 2nd international Conference on Big Data, Cloud and Applications, no. 61, pp. 685, 2017.

[10] WebHarvy: Intuitive Powerful Visual Web Scraper, https://www.webharvy.com/

[11] Chapman, P. et al. 2000. CRISP-DM 1.0 Step-by-step data mining guide. SPSS; 2000.

[12] D. Delen, "Extracting Knowledge from Published Literature Using RapidMiner", in Practical Text Mining and Statistical Analysis for Non-structured Text Data Applications, 2012.

[13] V. Kotu, B. Deshpande, "Getting Started with RapidMiner”, in Data Science (Second Edition), 2019.

[14] T. Verma, R. Renu, D.Gaur, "Tokenization and Filtering Process in RapidMiner", International Journal of Applied Information Systems, vol. 7, pp. 16-18, 2014.

[15] M, Porter, "An algorithm for suffix stripping”, Program: electronic library and information systems, vol. 14, no. 3, pp. 130-137, 1980.

[16] A. Jivani, “A Comparative Study of Stemming Algorithms”, Int. J. Comp. Tech. Appl., vol. 2, pp. 1930-1938, 2011.

[17] D. Delen, "Predicting Box Office Success of Motion Pictures with Text Mining", Practical Text Mining and Statistical Analysis for Non-structured Text Data Applications, 2012

[18] J.Yi, T. Nasukawa, R. Bunescu, W. Niblack. "Sentiment analyzer: Extracting sentiments about a given topic using natural language processing techniques", in Proceedings of the IEEE International Conference on Data Mining, 2013.

[19] A. Dabirian, J. Kietzmann, H. Diba, "A great place to work!? Understanding crowdsourced employer branding”, Business Horizon, vol. 60, no. 2, pp. 197-205, 2016.

[20] P. D. Hong, A. C. Le, "Exploiting multiple word embeddings and one-hot character vectors for aspect-based sentiment analysis", Int. J. Approx. Reason, vol. 103, pp. 1-10, 2018.

[21] K. Bafna, D. Toshniwal, "Feature Based Summarization of Customers' Reviews of Online Products", Procedia Computer Science, vol. 22 , pp. 142-151, 2013. 